\title{
Fenómenos de somatización en la población penitenciaria: diagnóstico y tratamiento
}

\author{
J García Campayo
}

Psiquiatra y Profesor Asociado.

Hospital Miguel Servet y Universidad de Zaragoza

\section{RESUMEN}

Los procesos fisiológicos diarios, el exceso de fatiga física, infecciones víricas subclínicas o determinadas circunstancias ambientales, son capaces de producir algún tipo de dolor o malestar físico en el $75 \%$ de los adultos en el plazo de una semana. Cuando, en contra de la opinión de su médico, el individuo atribuye estas molestias a una enfermedad orgánica y busca un diagnóstico y un tratamiento de forma persistente, hablamos de somatización. Trastornos depresivos y de ansiedad o incluso una verdadera alteración de la personalidad, están detrás de la mayoría de este tipo de trastornos.

La falta de estudios de prevalencia de este tipo de trastornos en prisión impide valorar adecuadamente la importancia de este fenómeno en las consultas de atención primaria de las prisiones, si bien cabe esperar un volumen similar de estos trastornos, sino mayor al encontrado en la población general, en torno al $15 \%$.

El manejo de este tipo de paciente es siempre conflictivo, complejo y emocionalmente muy exigente para el profesional sanitario.

Palabras Clave: Trastornos Somatoformes, Literatura de Revisión, Prisión.

\section{UNEXPLAINED PHSYICAL COMPLAINTS AMONGST PRISON INMATES: DIAGNOIS AND TREATMENT}

\begin{abstract}
Daily physiological processes, excess physical fatigue, subclinical viral infections and certain environmental situations can all cause some kind of physical pain or malaise in $75 \%$ of adults in a typical week. When the individual refuses to accept medical opinion and attributes the pain to a physical illness and persistently seeks diagnosis and treatment, then there is a possibility of an unexplained somatic problem. Depression, anxiety or even a personality disorder may be causative factors.

The lack of studies of this type of disorder in the prison environment makes it difficult to fully evaluate the importance of this phenomenon for primary health care consultation in prison, despite the fact that the number of presented unexplained somatic complaints may well be the same as in the community (around $15 \%$ ).

Management of this type of patient is always difficult, complex and emotionally demanding for the health care professional.
\end{abstract}

Key words: Somatoform Disorders, Review Literature, Prison.

Texto recibido: julio 2007

Texto aceptado: octubre 2007

\section{EL CONCEPTO DE SOMATIZACION: QUÉ ES Y QUÉ NO ES SOMATIZAR}

A) LA PERCEPCION DE LOS SINTOMAS SOMATICOS EN EL SER HUMANO: La experimentación de síntomas somáticos es un suceso muy habitual en el ser humano. De hecho, los estudios sobre el tema demuestran que el $75 \%$ de los adultos sanos padecen algún tipo de dolor o malestar físico en el plazo de una semana ${ }^{1}$. Las causas que pueden producir estos síntomas son tres ${ }^{1}: 1$.- Los procesos fisiológicos diarios, como los de respiración, digestión, 
circulación y las modificaciones hormonales del sistema endocrino. 2.- Circunstancias que no son enfermedad: Las principales son modificaciones en la dieta, exceso de fatiga física o infecciones víricas subclínicas pero que producen mínimas molestias. 3.- El medio ambiente: Factores ambientales como calor o frío extremo, exceso de luz, humedad o ruido, contaminación ambiental (humo de tabaco, por ejemplo), tormentas o cambios de clima, etc.

Es decir, la condición normal en el hombre es experimentar síntomas somáticos de forma frecuente y eso no constituye ninguna enfermedad psiquiátrica. Sólo debe hablarse de somatización, y catalogarse como un problema médico, cuando el individuo atribuye estas molestias a una enfermedad orgánica y busca un diagnóstico y un tratamiento médico de forma persistente, pese a las reafirmaciones de los médicos en el sentido de que no padece ninguna enfermedad física grave que esté produciendo los síntomas.

B) EL CONCEPTO DE SOMATIZACIÓN: El concepto de somatización siempre incluye tres componentes ${ }^{2}$ :

- EXPERIMENTAL: Expresa lo que el individuo percibe en relación a su cuerpo, sea dolor u otras manifestaciones corporales desacostumbradas, disfuncionales o de cambio de apariencia. Generalmente sólo es conocido por el propio individuo y es difícil de evaluar por el clínico, quien tiende a minusvalorarlo.

- COGNITIVO: Se basa en la interpretación que el sujeto realiza de estas percepciones y a qué las atribuye, es decir, el significado subjetivo que tienen para él. También incluye el proceso de toma de decisiones que surgen a partir de aquí. En el caso de los somatizadores, las sensaciones somáticas son interpretadas como una enfermedad amenazante o capaz de producir daño corporal y por eso busca la ayuda del médico. Esta convicción de riesgo para la salud persiste pese a las reafirmaciones médicas en sentido contrario.

- CONDUCTUAL: Incluye las acciones y la comunicación verbal y no verbal que sigue a dichas atribuciones. Típicamente, aunque no de forma invariable, el sujeto se decide a buscar consejo o tratamiento en la medicina o en otras alternativas paramédicas. No todos siguen este curso de acción: algunos se tratan a sí mismos, buscan el consejo de amigos o se dedican, exclusivamente, a quejarse ante familiares o personas no profesionales. Desde el punto de vista del sistema sanitario, los que buscan ayuda médica, sobre todo de forma persistente, representan un serio problema de salud comunitaria.

\section{C) ¿QUÉ ES UN PACIENTE "SOMATIZA- DOR"?}

Actualmente se acepta que paciente somatizador es aquél que cumple los denominados criterios de Bridges y Goldberg ${ }^{3}$ que son los que se resumen en la Tabla I.

1.- El paciente consulta por síntomas somáticos.

2.- El paciente atribuye los síntomas que padece a una enfermedad orgánica y no a un trastorno psiquiátrico.

3.- Existe un trastorno psiquiátrico diagnosticable con las clasificaciones psiquiátricas actuales.

4.- En opinión del médico, el tratamiento del trastorno psiquiátrico mejoraría los síntomas físicos.

Tabla I. Criterios de paciente somatizador ${ }^{3}$.

Aunque han existido críticas a esta definición, sobre todo al criterio número cuatro porque puede considerarse subjetivo ${ }^{4}$, es la más aceptada para conceptualizar qué es un paciente somatizador. En esta definición se incluyen, mayoritariamente, pacientes que cumplen criterios diagnósticos de trastornos depresivos, de ansiedad y de adaptación. Sólo menos del $10 \%$ cumplen criterios de trastornos somatomorfos ${ }^{5}$, los cuales constituyen la "punta del iceberg" de los fenómenos de somatización, porque representan las formas más crónicas e invalidantes.

Una clasificación importante dentro de los pacientes somatizadores tiene que ver con la evolución temporal ${ }^{3}:$ Se habla de somatizadores agudos o subagudos cuando la duración de los síntomas es menor o igual a 6 meses. Corresponden a los trastornos depresivos, de ansiedad y de adaptación que aparecen en forma de síntomas somáticos. La forma de presentación «somatizada» de estos tres grupos de enfermedades es tres veces más frecuente que la presentación mediante síntomas psicológicos ${ }^{3,5} \mathrm{y}$, por esta razón, algunos autores han propuesto la especificación en el próximo DSM, dentro del trastorno depresivo mayor, del epígrafe «presentación en forma de síntomas somáticos» ${ }^{6}$. Por el contrario, se consideran somatizadores crónicos cuando la enfermedad persiste más de 6 meses. Estos cuadros se identifican con los trastornos somatomorfos.

\section{LOS TRASTORNOS SOMATOMORFOS}

Los trastornos somatomorfos, según la cuarta versión del Manual diagnóstico y estadístico de los trastornos mentales (DSM-IV) de la Asociación Psiquiátrica Americana ${ }^{7}$, constituyen un grupo de enfermedades 
psiquiátricas que se caracterizan porque el paciente presenta síntomas corporales que sugieren un trastorno físico. Sin embargo, no existe ninguna causa orgánica demostrable o mecanismo fisiopatológico conocido que los explique completamente. Por otra parte, existe una presunción razonable de que dichos síntomas están asociados a factores psicológicos o al estrés.

La categoría de los trastornos somatomorfos se creó en 1980, cuando se describió por primera vez en el DSM-III, y su validez conceptual ha sido muy discutida. El principal argumento a favor de su utilización es su incuestionable utilidad clínica. Sin embargo, existen una serie de dificultades nosológicas no resueltas que son las siguientes:

A.- COMORBILIDAD EN EL EJE I: La comorbilidad en el Eje I de los trastornos somatomorfos es muy elevada. Por ejemplo, en el caso del trastorno por somatización, nuestro grupo encontró en población española comorbilidad en el $85 \%$ de estos pacientes (8), con predominio de trastornos depresivos y de ansiedad, como se refleja en la Tabla II.

\begin{tabular}{lc}
\hline $\begin{array}{l}\text { Diagnóstico psiquiátrico } \\
\text { asociado (Eje I) }\end{array}$ & Porcentaje \\
\hline Distimia & $40 \%$ \\
\hline Trastorno de ansiedad generalizada & $25.7 \%$ \\
\hline Trastorno por angustia & $22,8 \%$ \\
\hline Abuso de analgésicos & $20 \%$ \\
\hline Agorafobia & $17,1 \%$ \\
\hline Depresión mayor & $5,7 \%$ \\
\hline Depresión no especificada & $5,7 \%$ \\
\hline Otros & $14,2 \%$ \\
\hline
\end{tabular}

Tabla II. Comorbilidad psiquiátrica del trastorno por somatización en población española ${ }^{8}$.
B.- COMORBILIDAD EN EL EJE II: También la comorbilidad psiquiátrica en el Eje II de los trastornos somatomorfos es muy elevada, sobrepasando el 50\% en la mayoría de las categorías de esta sección, como resumimos en la Tabla III. Este hecho, ha llevado a algunos autores a recomendar su inclusión dentro del Eje II, porque consideran que los trastornos somatomorfos, más que trastornos psiquiátricos, constituyen una alteración de la personalidad del individuo?.

\begin{tabular}{lc}
\hline Trastorno & Prevalencia \\
\hline Trastorno por somatización & $72 \%$ \\
\hline Hipocondría & $63 \%$ \\
\hline Trastorno por dolor & $59 \%$ \\
\hline Trastorno dismórfico corporal & $65 \%$ \\
\hline
\end{tabular}

Tabla III. Comorbilidad en el Eje II de los trastornos somatomorfos?.

C.- CRITERIOS DIAGNÓSTICOS DIFERENTES EN LAS DOS PRINCIPALES CLASIFICACIONES PSIQUIÁTRICAS: Existen importantes diferencias entre las dos principales clasificaciones psiquiátricas, la Clasificación Internacional de Enfermedades $10^{a}$ edición (CIE-10) de la Organización Mundial de la Salud ${ }^{10}$ y el DSM-IV ${ }^{20}$, al describir el grupo de los trastornos somatomorfos, como resumimos en la Tabla IV. La principal consecuencia de este hecho, es que los índice de concordancia, cuando comparamos los trastornos somatomorfos, como por ejemplo el trastorno por somatización, diagnosticado con la CIE-10 y con el DSM-IV, son tan bajos (kap$\mathrm{pa}=0,53)$ que impide comparar estudios de investigación en los que se hayan utilizado ambos criterios $^{11}$.

\begin{tabular}{ll}
\hline CIE-10 & DSM-IV \\
\hline F 45.0 Trastorno por somatización. & Trastorno por somatización. \\
\hline F 45.1 Trastorno somatomorfo indiferenciado & Trastorno somatomorfo indiferenciado. \\
\hline F 45.2 Trastorno hipocondríaco & Hipocondría \\
\hline F 45.3 Disfunción vegetativa somatomorfa & Incluida en el T. S. indiferenciado \\
\hline F 45.4 Trastorno por dolor somatomorfo & Trastorno por dolor \\
\hline F 45.8 Otros trastornos somatomorfos & No existe \\
\hline F 45.9 Trastorno somatomorfo sin especificar & Trastorno somatomorfo no especificado \\
\hline Incluido en F 44. Trastorno disociativo & Trastorno por conversión \\
\hline Incluido en F 45.2 Hipocondría & Trastorno dismórfico corporal \\
\hline
\end{tabular}

Tabla IV. Clasificación de los trastornos somatomorfos en la CIE-10 y en el DSM-IV con sus correspondencias relativas. 
Las principales diferencias entre las dos clasificaciones son:

1.- Los trastornos conversivos se incluyen dentro de los trastornos somatomorfos en el DSM-IV pero la CIE-10 prefiere agruparlo junto a los trastornos disociativos porque, de esta forma, conserva el nicho conceptual de la histeria clásica.

2.- La CIE-10 incluye una categoría denominada disfunción vegetativa somatomorfa que no existe en el DSM-IV y que habría que encuadrarlo dentro del trastorno somatomorfo indiferenciado.

3.- La CIE-10 no otorga categoría conceptual distintiva al trastorno dismórfico corporal y lo considera un subtipo de hipocondría, mientras que para la DSM constituye una entidad distinta.

4.- La CIE-10 acepta un síndrome de fatiga crónica en un capítulo aparte, trastorno que no se acepta en el DSM-IV.

Todas estas circunstancias han determinado que, en la actualidad, la sección de los trastornos somatomorfos sea una de las más criticadas de las clasificaciones psiquiátricas.

\section{IMPORTANCIA CLÍNICA DE LOS TRASTORNOS SOMATOMORFOS}

La importancia clínica de los trastornos somatomorfos para el sistema sanitario de cualquier país depende de los siguientes cuatro factores:

1.- ELEVADA PREVALENCIA: En el estudio ECA, la prevalencia de los trastornos somatomorfos en la población general variaba desde el 0,38\% (12) al $4,4 \%{ }^{13}$ dependiendo del número de síntomas requeridos. En atención primaria, y según datos de nuestro país ${ }^{14}$, se calcula que más del $1 \%$ de los pacientes que consultan al médico de familia por un nuevo episodio de enfermedad son diagnosticados de trastorno de somatización. También dentro del hospital este grupo de enfermedades es importante, ya que el $15 \%$ de los pacientes vistos en los servicios de Psiquiatría de Enlace e Interconsulta presentan trastornos somatomorfos ${ }^{15}$.

2.- ESCASA CALIDAD DE VIDA: Pese a que la incapacidad que producen los trastornos somatomorfos es similar a la que se asocia a otros trastornos psiquiátricos (16), la calidad de vida de estos pacientes es más pobre que la de otros pacientes con trastornos crónicos como SIDA, cancer, diabetes, enfermedad de Parkinson o cardiopatías ${ }^{17}$. De hecho, son los pacientes que relatan peor calidad de vida de todas las enfermedades orgánicas y psiquiátricas estudiadas ${ }^{18}$.
3.- ELEVADOS COSTES SANITARIOS: La somatización supone el 10\% del gasto sanitario total de cualquier país desarrollado (19). Dicho coste no incluye los gastos de invalidez o de las bajas laborales. El coste anual medio por paciente con trastorno de somatización se ha calculado en unos 1.000 dólares $^{20}$.

\section{4.- DIFÍCIL MANEJO POR PARTE DE LOS} PROFESIONALES SANITARIOS: Los somatizadores se consideran uno de los pacientes más difíciles en su relación con los profesionales sanitarios. La razón es que suelen estar descontentos con cualquier tratamiento que se les ofrece y solicitan continuas e injustificadas demandas de ayuda médica ${ }^{21}$. La consecuencia es que una elevada proporción de médicos rechaza tratar estos pacientes por los sentimientos de frustración e ira que tienden a producir en el profesional ${ }^{22}$.

\section{TRASTORNO SOMATOMORFOS EN ENTORNOS CARCELARIOS}

No existen estudios específicos sobre este tema como se confirma en la revisión bibliográfica realizada en Medline y Embase desde 1966 con las palabras clave "somatization"/somatoform disorders" and “prison”/“jail”. Podemos deducir la prevalencia de trastornos somatomorfos en base a los estudios sobre patología psiquiátrica en general realizados en este entorno $(23,24)$ y, sobre estos datos, sabemos que la prevalencia de trastornos somatomorfos en esta población es similar a la encontrada en atención primaria, es decir, entre el 5-15\%.

Para acercarnos lo máximo posible a las características de los pacientes somatizadores en entornos carcelarios, hemos analizado algunos de los trastornos psiquiátricos más frecuentes en esta población (abuso de sustancias y trastorno de personalidad), y su asociación con los trastornos somatomorfos.

a) TRASTORNOS DE PERSONALIDAD: El trastorno de personalidad constituye la patología psiquiátrica más prevalente en las prisiones, con cifras entre $50-75 \%$, como se ha confirmado en los estudios referidos $^{23,24}$. Ya hemos comentado anteriormente la importante comorbilidad existente entre trastornos de personalidad y trastornos somatomorfos. Sin embargo, no existen demasiados estudios sobre los subtipos específicos de trastornos de personalidad que se asocian a trastornos somatomorfos. Nuestro grupo ha publicado recientemente un estudio controlado sobre el tema, con una amplia muestra $(\mathrm{N}=70)$ (25). En nuestro estudio, los trastornos de personalidad específicos con mayor odds-ratio en relación a la población normal fue- 
ron el trastorno de personalidad paranoide $(\mathrm{OR}=9,2$; 95\% IC=1.9-43); obsesivo-compulsivo ( $\mathrm{OR}=6.2 ; 95 \%$ $\mathrm{IC}=1.2-53.6)$ e histriónico $(\mathrm{OR}=3.6 ; 95 \% \mathrm{IC}=0.9-$ 13.9). Estos serían los trastornos más intensamente asociados y por tanto algunos de los que más veríamos también en población carcelaria. Lógicamente, habría que añadir el trastorno límite de personalidad (que es el más frecuente en cualquier población incluso en sujetos sin patología psiquiátrica en el Eje I) y el trastorno de personalidad antisocial, que es el más intensamente asociado a la situación de privación de libertad.

b) ABUSO DE SUSTANCIAS: La comorbilidad entre abuso de sustancias y somatización varía, lógicamente, en dependencia del nivel asistencial donde se estudie, como resumimos en la Tabla V.

\begin{tabular}{lcc}
\hline $\begin{array}{l}\text { Nivel } \\
\text { asistencial }\end{array}$ & $\begin{array}{c}\text { Abuso/ } \\
\text { dependencia } \\
\text { drogas }\end{array}$ & $\begin{array}{c}\text { Abuso/ } \\
\text { dependencia } \\
\text { alcohol }\end{array}$ \\
\hline Comunidad & No hay datos & $17,7 \%$ \\
\hline Atención primaria & $4,9 \%$ & $21,0 \%$ \\
\hline Consultas externas & $23,1 \%$ & $16,7 \%$ \\
\hline Hospitalización & $31,2 \%$ & $43.4 \%$ \\
\hline
\end{tabular}

Tabla V. Comobilidad trastorno de somatización y abuso de sustancias según nivel asistencial ${ }^{26}$.

Sin embargo, cuando se compara no la prevalencia absoluta, sino la prevalencia relativa, la comorbilidad abuso/dependencia de alcohol/substancias es la misma que la de la población general. Cuando lo analizamos desde la perspectiva inversa, es decir, el porcentaje de pacientes con abuso/dependencia de alcohol/substancias que presentan trastornos somatomorfos, las cifras se sitúan alrededor del 4,6\% que también son similares a los de la población general ${ }^{26}$.

No obstante, los pacientes con comorbilidad de somatización y abuso/dependencia de alcohol y/o sustancias presentan unas características específi$\mathrm{cas}^{26}$, que resumimos a continuación y que van a encontrarse en los pacientes en entornos carcelarios:

Hiperconsultante: Se definen como hospitalizados al menos 10 veces en 8 años o que consultan al médico más de 1 vez cada 15 días (aunque existen múltiples definiciones diferentes y no existe un acuerdo total en este término).

Escasa adherencia al tratamiento: Ya hemos visto en el punto anterior que muestran una mayor tendencia a buscar tratamiento, pero en este punto insistimos que también muestran una mayor facilidad para abandonarlo.
Estilo demandante y manipulador: Su manejo de la relación médico-paciente (pero sería igual en todas las demás interacciones, p.ej.: con los funcionarios de prisiones) se encuentran moduladas por este patrón.

Los fenómenos de somatización, manipulación, defensas inmaduras y aumento de la patología psiquátrica en general se ha observado también en poblaciones específicas como adolescentes internados en correccionales ${ }^{27}$ y en madres en prisión separadas de sus hijos $^{28}$. Para este perfil de funcionamiento se supone que existirían factores causales bioquímicos, de personalidad y de desarrollo educativo e interaccional con el entorno a lo largo de su biografía. Todo esto conllevaría una baja tolerancia a la frustración y una búsqueda constante de estímulos, de forma que se encuentren en la situación en que se encuentren, siempre van a buscar el cambio. Lógicamente, esto se asocia a un peor pronóstico.

\section{TRATAMIENTO DE LOS TRASTORNOS SOMATOMORFOS EN LAS INSTITUCIONES PENITENCIARIAS}

\section{a) INTRODUCCION}

Como parece obvio, no puede ser muy diferente del que se propone habitualmente para atención primaria. Existen una serie de recomendaciones terapéuticas muy conocidas, que ha propuesto el grupo de Smith $(29,30)$ y que resumimos en la Tabla VI.

1.- El paciente debe ser atendido por un solo médico: su médico de familia.

2.- Planificar citas regulares cada 4-6 semanas como máximo.

3.- Realizar consultas breves que sean posibles en la agenda del generalista.

4.- Explorar siempre la zona de la que se queja el paciente.

5.- Buscar signos. Los síntomas deben ser interpretados en un contexto comunicacional.

6.- Evitar tests diagnósticos que no sean imprescindibles para evitar yatrogenia y gasto sanitario. Deben solicitarse basados en los signos, no en los síntomas.

7.- Evitar diagnósticos espúreos y tratamientos innecesarios.

8.- Tranquilizar y reasegurar.

9.- Derivar adecuadamente a los servicios psiquiátricos.

Tabla VI. Normas de buena práctica clínica en atención primaria con los pacientes con trastornos somatomorfos. 
La mayor dificultad para llevar a cabo estos programas es que los somatizadores crónicos producen sentimientos de frustración e ira y provocan un gran rechazo por parte de los profesionales sanitarios. De hecho, cuando se realizan encuestas a los médicos de familia, el 70\% rechaza ofrecer cualquier tipo de apoyo psicológico a los somatizadores aunque se les entrene específicamente para ello (31-33). Estos sentimientos se incrementan en los entornos carcelarios, en los que los individuos muestran las características disfuncionales que hemos comentado anteriormente.

b) TRATAMIENTO FARMACOLÓGICO: Dado que no existe un tratamiento etiológico de la enfermedad, el abordaje tendrá que ser sintomático. Los principales síntomas que presentan estos pacientes son los de la esfera psiquiátrica (depresión, ansiedad e insomnio) y los síntomas somáticos (con el dolor de diferentes partes del cuerpo como principal referente). Por esta razón, dividimos el tratamiento en estos dos epígrafes:

- SINTOMAS PSIQUIÁTRICOS: Un error común en atención primaria es no usar antidepresivos (sustituyéndolos por benzodiacepinas aunque exista depresión), o pautar antidepresivos a dosis bajas. Ambos deben evitarse: las benzodiacepinas, que presentan un cierto riesgo de adicción en la población general, estarían específicamente contraindicadas en los pacientes penitenciarios por su tendencia a la habituación. Por ello, si existe ansiedad se recomienda el uso de antidepresivos serotoninérgicos o duales con efecto sedante como duloxetina (60-120 mg/día) o mirtazapina (30-60 mg/día), o fármacos ansiolíticos no benzodiacepínicos como pregabalina (100-200 $\mathrm{mg} /$ día es la dosis ansiolítica habitual). Si hay insomnio también se recomiendan antidepresivos que actúan sobre la serotonina como la trazodona (100 $\mathrm{mg} /$ día en toma nocturna) mejor que hipnóticos. Si hay que usarlos, mejor los que no son benzodiacepínicos como zolpidem o zopiclona. Por último, si hay depresión, puede usarse cualquier antidepresivo a dosis adecuadas.

- SINTOMAS SOMÁTICOS/DOLOR: Para los síntomas somáticos en general y, concretamente, para el dolor, son especialmente efectivos los antidepresivos duales como duloxetina (60-120 mg/día), venlafaxina (150-225 mg/día) y mirtazapina (30-60 $\mathrm{mg} /$ día). Aquí no estarían indicados los antidepresivos sertoninerégicos porque mejorarían la depresión pero no tanto el dolor. Si es necesario, suelen asociarse antiepilépticos con efecto analgésico como carbamacepina (400-1.200 mg/día), oxicarbamacepina (600-1.800 mg/día) pero, sobre todo, gabapentina
(600-1.200 mg/día) y pregabalina (150-600 mg/día es la dosis analgésica, como se ve mayor que la ansiolítica). Pueden asociarse antiepilépticos y antidepresivos sin demasiados problemas. Además de esto, ocasionalmente son necesarios otros analgésicos a dosis bajas y poco frecuentes, teniendo siempre en cuenta la tendencia a la adicción a analgésicos de la población penitenciaria.

c) TRATAMIENTO PSICOLÓGICO: En cuanto al tratamiento psicológico, a nivel de salud mental se han ensayado distintos tipos de tratamientos como la psicoterapia cognitivo-conductual tanto individual como en grupo (la más empleada y efectiva), la psicoterapia dinámica y, excepcionalmente, otras psicoterapias tipo Gestalt o familiar. No vamos a describirlas aquí porque corresponden al ámbito especializado por su consumo de tiempo y por la formación requerida para su administración.

Sin embargo, si que insistiremos en el MODELO EDUCATIVO/DE APOYO, descrito por Smith ${ }^{29,30}$, que no requiere una formación especial y que se recomienda en primaria pudiendo adecuarse perfectamente al entorno de la prisión. Se realiza en grupos de 8-10 pacientes y su objetivo es promover la ayuda mutua entre los miembros, compartir métodos de afrontamiento de los síntomas físicos, aumentar la capacidad de los pacientes para expresar emociones y disfrutar con la experiencia. Se estructura en forma de 8 sesiones de $90 \mathrm{mn}$. y fomenta un ambiente de tipo educativo, no psicoterapéutico, porque es mucho mejor aceptado por los enfermos. Esto es aún más cierto en la prisión. En cada sesión se exponen temas como formas de afrontar las molestias físicas, la asertividad en las relaciones interpersonales, cómo adquirir más control en la vida diaria y cómo resolver problemas. Existen estudios que muestran que los pacientes que asisten al grupo muestran mejor salud psicológica, mejor funcionamiento físico y mejor salud general percibida por el individuo ${ }^{29,30}$.

\section{CONCLUSIÓN}

Como se ve, nos encontramos ante un grupo de pacientes muy prevalente en todos los niveles sanitarios, también en la población penitenciaria, y que presentan un gran desafío al sistema de salud por su elevado coste y dificultad de manejo. En este artículo nos hemos centrado en la comprensión clínica de este fenómeno y en analizar las características específicas que pudiesen presentar en el entorno concreto de las prisiones, intentando facilitar así su diagnóstico. 
De la misma forma, hemos resumido algunas estrategias terapéuticas sencillas y eficaces accesibles a los profesionales sanitarios que trabajan en este medio.

\section{REFERENCIAS BIBLIOGRÁFICAS}

1. White KL, Williams TF, Greenberg BG. The ecology of medical care. N Eng J Med 1961; 265: 885892.

2. Lipowski ZJ. Somatization: the concept and its clinical application. Am J Psychiatry 1988; 145: 1358-68.

3. Bridges KW, Goldberg DP. Somatic presentation of DSM-III psychiatric disorders in primary care. J Psychosomatic Res 1985; 29: 563-69.

4. García-Campayo J, Sanz Carrillo C. Somatisers and psychologisers in primary care: a review. International Journal of Psychiatry in Medicine 1999; 29: 337-45.

5. Lobo A, García-Campayo J, Campos R, PérezEcheverría MJ, Marcos G. Somatization in primary care in Spain: I. Estimates of prevalence. British Journal of Psychiatry 1996; 168: 344-48.

6. Rief W, Hiller W. Somatization: future pespectives on a common phenomenon. Journal of Psychosomatic Research 1998; 5: 529-36.

7. Asociación Psiquiátrica Americana. Manual diagnóstico y estadístico de los trastornos mentales. Cuarta edición (DSM-IV). Barcelona: Masson, 1995.

8. García-Campayo J, Sanz Carrillo C, Pérez-Echeverría MJ, Campos R, Lobo A. Trastorno por somatización en atención primaria: aspectos clínicos diferenciales. Med Clin (Barc) 1995; 105: 728-733.

9. Bass C, Murphy M. Somatoform and personality disorders: syndromal comorbidity and overlapping developmental pathways. J Psychosom Res 1995; 39: 403-428.

10. Organización Mundial de la Salud. Décima revisión de la Clasificación Internacional de Enfermedades (CIE 10). Trastornos mentales y del comportamiento. Madrid: Meditor, 1994.

11. Rief W, Heuser J, Mayrhuber E, Stelzer I, Hiller W, Fichter MM. The classification of multiple somatoform symptoms. J Nerv Ment Dis 1996; 184: 680-687.

12. Swartz MS, Blazer DG, George LK, Laderman R. Somatization disorder in a community population. Am J Psychiatry 1986; 143: 1403-1408.

13. Escobar JI, Rubio-Stipec M, Canino G, Karno M. Somatic symptom index (SSI): a new abridged somatization construct. Prevalence and epidemiolo- gical correlates in two large community samples. J Nerv Ment Dis 1989; 177: 140-146.

14. García-Campayo J, Campos R, Marcos G, PérezEcheverría MJ, Lobo A. Somatization in primary care in Spain. II. Differences between somatisers and psychologisers. Br J Psychiatry 1996; 168: 348-353.

15. De Leon J, Saiz-Ruiz J, Chinchilla A. Why do some psychiatric patients somatize? Acta Psychiatr Scand 1987; 76: 203-209

16. Hiller W, Rief W, Fichter MM. How disabled are patients with somatoform disorders? Gen Hosp Psychiatry 1997; 19: 432-438.

17. Kroyman R, Hiller W, Rief W, Fichter M. Somatization and quality of life. Paper presented at Somatoform Disorders Symposium. Prien, Germany. 23-25 $5^{\text {th }}$ February 1997.

18. Barsky AJ, Wyshak G, Klerman GI. Medical and psychiatric determinants of outpatient medical utilization. Medical Care 1986; 24: 548-563.

19. Barsky AJ, Klerman GL. Overview. Hypochondriasis, bodily complaints and somatic styles. Am J Psychiatry 1983; 140: 273-283.

20. Smith JR G, Rost K, Kashner M. A trial of the effect of a standardized psychiatric consultation on health outcomes and costs in somatizing patients. Arch Gen Psychiatry 1995; 52: 238-243.

21. Lipsitt DR. Medical and psychological characteristics of "crocks". Psychiatry in Medicine 1970; 1: 15-25.

22. García-Campayo J, Sanz-Carrillo C, Yoldi A, Lopez-Aylon R. Management of somatizers in primary care: are family doctors motivated? Austr NZ J Psychiatry 1998; 32: 528-533.

23. Brinded PM, Simpson AI, Ladlaw Tm, et al. Prevalence of psychiatric disorders in New Zealand prisons: a nacional study. Austr NZ J Psychiatry 2001; 35: 166-73.

24. Birmingham L, Mason D, Grubin D. Prevalence of mental disorder in remand prisons: consecutive case study. BMJ 1996; 313: 1521-24.

25. Garcia Campayo J, Alda M, Sobradiel N, Olivan B, Pascual A. Personalities disorders in somatization disorder patients: a controlled study in Spain. J Psychosom Res 2007; 62: 675-80.

26. Sanz Carrillo C, Garcia Campayo J. Manejo terapéutico de los trastornos psicosomáticos y abuso de sustancias. En: Trastornos psiquiátricos y abuso de sustancias. G. Rubio, F. López-Muñoz, C. Alamo, J. Santo-Domingo (eds). Madrid: Panamericana, 2002.

27. Steiner H, Garcia IG, Matthews Z. Posttraumatic stress disorder in incarcerated juvenile delin- 
quents. J Am Acad Child Adolesc Psychiatry 1997; 36:357-65.

28. Houck KD, Lopez AB. The relationship of parenting stress to adjustment among mothers in prison. Am J Orthopsychiatry 2002; 72:548-58.

29. Smith GR. Somatization disorder in the medical setting. Washington: American Psychiatric Press, 1991.

30. Smith JR GR, Rost K, Kashner M. A trial of the effect of a standardized psychiatric consultation on health outcomes and costs in somatizing patients. Arch Gen Psychiatry 1995; 52: 238-243.

31. García-Campayo J, Sanz-Carrillo C, Montón C. Dificultades en la relación médico-paciente en somatizadores (I): expectativas del paciente. Med Clin (Barc) 1999; 112: 102-105.
32. Sanz-Carrillo C, García-Campayo J, Montón C. Dificultades en la relación médico-paciente en somatizadores (II): reacciones del profesional y tipos de relación. Med Clin (Barc) 1999; 112: 147150.

33. García-Campayo J, Sanz Carrillo C, Yoldi A, López Aylon R. Management of somatizers in primary care: are family doctors motivated? Austr NZ J Psychiatry 1998; 32: 528-33.

\section{CORRESPONDENCIA}

Talleres Editoriales Cometa, S.A.

Ctra. Castellón, km. 3,400.

50013 Zaragoza. 